\title{
New Oscillation Conditions for Second Order Half-Linear Advanced Difference Equations
}

\author{
P. Dinakar \\ Department of Mathematics, \\ Presidency College, University of Madras, Chennai - 600005, India \\ E-mail: dinakarraj@gmail.com \\ S. Selvarangam \\ Department of Mathematics, \\ Presidency College, University of Madras, Chennai - 600005, India \\ E-mail: selvarangam.9962@gmail.com \\ E. Thandapani \\ Ramanujan Institute for Advanced Study in Mathematics, \\ University of Madras, Chennai - 600005, India \\ Corresponding author: ethandapani@yahoo.co.in
}

(Received March 25, 2019; Accepted August 15, 2019)

\begin{abstract}
This paper aims to establish adequate conditions that are intended for the oscillation of every solution of the second order advanced type half-linear difference equations with noncanonical form. Initially, we derive a sufficient condition that ensures all solutions of the studied equation are either oscillatory or tending to zero. Secondly, we obtain a criteria for the oscillation of all solutions of the studied equation. These criteria are obtained by using Riccati transformation and summation averaging method. The results established in this paper in essence complement, extend and enhance the existing outcomes recorded in the literature. The improvement of our main results are illustrated through three examples.
\end{abstract}

Keywords- Asymptotic behavior, Half-linear difference equation, Oscillation, Second order.

\section{Introduction}

The second order half-linear difference equations of the semblance

$\Delta\left(a_{n}\left(\Delta y_{n}\right)^{\alpha}\right)+q_{n} y_{\sigma(n)}^{\alpha}=0, \quad n \geq n_{0}$,

where $\alpha>0$, whose solutions oscillate is studied subject to the conditions:

(i) $\left\{a_{n}\right\}$ is a positive real sequence and $\{\sigma(n)\}$ is such that $\sigma(n)>n+1$ and $\Delta(\sigma(n)) \geq 0$ for all $n \geq n_{0}$;

(ii) $\left\{q_{n}\right\}$ is a nonnegative real sequence and it is not vanishing identically for many values of $n \geq n_{0}$.

A nontrivial real valued sequence $\left\{y_{n}\right\}$ satisfying (1) for all $n \geq n_{0}$ is called a solution of (1) and regarding only those solutions that exist for $n \geq N \geq n_{0}$ satisfying 
International Journal of Mathematical, Engineering and Management Sciences

Vol. 4, No. 6, 1459-1470, 2019

https://dx.doi.org/10.33889/IJMEMS.2019.4.6-115

$$
\sup \left\{\left|x_{n}\right|: n \geq N_{1}\right\}>0 \text { for any } \quad N_{1} \geq N
$$

A solution of (1) is oscillatory if it is neither eventually negative nor eventually positive, else termed as nonoscillatory. If all solutions of (1) oscillate, then it is regarded as oscillatory.

Adhering to Trench (1973), (1) is canonical if

$$
\sum_{t=n_{0}}^{n-1} a_{t}^{-\frac{1}{\alpha}} \rightarrow \infty \quad \text { as } \quad n \rightarrow \infty \text {. }
$$

Conversely, (1) is in noncanonical form if

$$
\sum_{t=n_{0}}^{\infty} a_{t}^{-\frac{1}{\alpha}}<\infty \text {. }
$$

Difference equations with advanced argument finds applications in the progression of growth rate which not only rely on the current, but extends to the near future. Inducing an advanced argument persuades the subsequent actions that are immediately accessible and helpful for decision making. Economic crisis and dynamics of population for instance are the phenomenal complications, contemplated to manifest (Elsgolts and Norkin, 1973; Agarwal, 2000).

A very great attention were received recently for the oscillatory and asymptotic behavior of solutions of difference equation (Agarwal, 2000; Agarwal et al., 2005). Though there was much investigation done on delay difference equations, meager studies were devoted to equations having advanced arguments (Zhang and Cheng, 1995; Zhang and Zhang, 1999; Thandapani et al., 2001; Ping and Han, 2003; Agarwal et al., 2005; Ocalan and Akin, 2007; Arul and Ayyapan, 2013; Selvarangam et al., 2016; Wu et al., 2016).

Ping and Han (2003) considered the following equation

$$
\Delta\left(a_{n}\left(\Delta y_{n}\right)\right)+p_{n} y_{n}+q_{n} y_{\sigma(n)}=0, \quad n \geq n_{0},
$$

and obtained some sufficient conditions for the oscillation of all solutions of equation (4) when it is in canonical form.

Ocalan and Akin (2007) considered the following equation

$\Delta y_{n}+p_{n} y_{n+k}=0, \quad n \geq n_{0}$,

and established several sufficient conditions for the oscillation of all solutions of equation (5)

Zhang and $\mathrm{Li}$ (1998) considered the following equation

$$
\Delta\left(a_{n}\left(\Delta y_{n}\right)\right)+q_{n} y_{\sigma(n)}=0, \quad n \geq n_{0},
$$

and derived several oscilltion criteria through Riccati equation when equation (6) is in canonical form. 
International Journal of Mathematical, Engineering and Management Sciences

Vol. 4, No. 6, 1459-1470, 2019

https://dx.doi.org/10.33889/IJMEMS.2019.4.6-115

Arul and Ayyappan (2013) considered the following equation

$\Delta\left(a_{n} \Delta\left(y_{n}+p_{n} y_{n+k}\right)\right)+q_{n} y_{n+l}+v_{n} y_{n+m}^{\alpha}=0, \quad n \geq n_{0}$,

and obtained conditions for the oscillation of all solutions of equation (7) when it is in canonical form.

From the above review of literature one can see that all the oscillation results established for the advanced type difference equations are linear and in canonical form. Therefore in this paper we obtain oscillation criteria for second order advanced difference equation with noncanonical form since such equations include Euler-type difference equations as a special case.

\section{Main Results}

Henceforth assume that (3) holds. Define

$$
A(n)=\sum_{s=n}^{\infty} a_{s}^{\frac{-1}{\alpha}}
$$

Let us consider only the positive solutions of (1), for if $\left\{y_{n}\right\}$ satisfies (1), then $\left\{-y_{n}\right\}$ also does.

\section{Lemma 1 Assume that}

$$
\sum_{n=n_{0}}^{\infty} q_{n}=\infty
$$

Further, assume that (1) has a positive solution $\left\{y_{n}\right\}$ for all $n \geq n_{1} \geq n_{0}$. Then

$$
y_{n}>0, \quad \Delta y_{n}<0, \quad \Delta\left(a_{n}\left(\Delta y_{n}\right)^{\alpha}\right) \leq 0
$$

for $n \geq n_{1}$. Moreover $\left\{\frac{y_{n}}{A(n)}\right\}$ is nondecreasing for $n \geq n_{1}$.

Proof: Let $\left\{y_{n}\right\}$ be a positive solution of (1) for $n \geq n_{1}$. From (1), we have

$$
\Delta\left(a_{n}\left(\Delta y_{n}\right)^{\alpha}\right)=-q_{n} y_{\sigma(n)}^{\alpha} \leq 0 .
$$

This implies that, $\left\{\Delta y_{n}\right\}$ is either positive or negative eventually. To the contrary suppose that (8) holds, and there is a $n_{2} \geq n_{1}$ such that $\Delta y_{n}>0$ for $n \geq n_{2}$. Define

$$
w_{n}=\frac{a_{n}\left(\Delta y_{n}\right)^{\alpha}}{y_{\sigma(n)}^{\alpha}}>0, \quad n \geq n_{2},
$$

and therefore $w_{n}>0$ and

$$
\Delta w_{n}=-q_{n}-\frac{a_{n+1}\left(\Delta y_{n+1}\right)^{\alpha}}{y_{\sigma(n+1)}^{\alpha} y_{\sigma(n)}^{\alpha}} \Delta y_{\sigma(n)}^{\alpha} \leq-q_{n} .
$$


International Journal of Mathematical, Engineering and Management Sciences

Vol. 4, No. 6, 1459-1470, 2019

https://dx.doi.org/10.33889/IJMEMS.2019.4.6-115

Summing (10) from $n_{2}$ to $n-1$, results in

$$
w_{n} \leq w_{n_{2}}-\sum_{s=n_{2}}^{n-1} q_{s} .
$$

In view of (8), it is evident that the last inequality contradicts the positivity of $\left\{w_{n}\right\}$. This justifies that $\Delta y_{n}>0$ is not possible implying that $\left\{y_{n}\right\}$ satisfies (9) for all $n \geq n_{1}$.

From the monotonicity of $a_{n}^{\frac{1}{\alpha}} \Delta y_{n}$ that for $l \geq n$,

$$
y_{n} \geq-\sum_{s=n}^{l} a_{s}^{\frac{-1}{\alpha}} a_{s}^{1 / \alpha} \Delta y_{s} \geq-a_{n}^{\frac{1}{\alpha}} \Delta y_{n} \sum_{s=n}^{l} a_{s}^{\frac{-1}{\alpha}} .
$$

Letting $l \rightarrow \infty$, we see that

$y_{n} \geq-a_{n}^{\frac{1}{\alpha}} \Delta y_{n} A(n)$.

Now we have from (11)

$$
\begin{aligned}
& \Delta\left(\frac{y_{n}}{A(n)}\right)=\frac{a_{n}^{1 / \alpha} A(n) \Delta y_{n}+y_{n}}{a_{n}^{\frac{1}{\alpha}} A(n) A(n+1)} \geq 0 . \\
& \text { Hence }\left\{\frac{y_{n}}{A(n)}\right\} \text { is nondecreasing, which completes the proof. }
\end{aligned}
$$

Theorem 2 Suppose that

$$
\sum_{n=n_{0}}^{\infty}\left(\frac{1}{a_{n}} \sum_{s=n_{0}}^{n-1} q_{s}\right)^{\frac{1}{\alpha}}=\infty \text {. }
$$

Furthermore, if (1) has a positive solution $\left\{y_{n}\right\}$ for all $n \geq n_{1} \geq n_{0}$, then $\left\{y_{n}\right\}$ satisfies (9) for all $n \geq n_{1}$ and

$\lim _{n \rightarrow \infty} y_{n}=0$.

Proof: Let $\left\{y_{n}\right\}$ be a positive solution of (1) for $n \geq n_{1}$. From (13) and (3), the unboundedness of $\sum_{s=n_{0}}^{n-1} q_{s}$ is evident, implying (8) holds. By Lemma 1, $\left\{y_{n}\right\}$ satisfies (9) for $n \geq n_{1}$.

Since $\left\{y_{n}\right\}$ is positive decreasing, there is $M \geq 0$ such that $\lim _{n \rightarrow \infty} y_{n}=M$. Assume $M>0$. Then there is an integer $n_{2} \geq n_{1}$ such that 
International Journal of Mathematical, Engineering and Management Sciences

Vol. 4, No. 6, 1459-1470, 2019

https://dx.doi.org/10.33889/IJMEMS.2019.4.6-115

$$
-\Delta\left(a_{n}\left(\Delta y_{n}\right)^{\alpha}\right)=q_{n} y_{\sigma(n)}^{\alpha} \geq M^{\alpha} q_{n}, \quad n \geq n_{2} .
$$

Taking summation from $n_{2}$ to $n-1$, in the last inequality, leads to

$$
-a_{n}\left(\Delta y_{n}\right)^{\alpha} \geq a_{n_{2}}\left(\Delta y_{n_{2}}\right)^{\alpha}+M^{\alpha} \sum_{s=n_{2}}^{n-1} q_{s}
$$

that is,

$$
-\Delta y_{n} \geq M\left(\frac{1}{a_{n}} \sum_{s=n_{2}}^{n-1} q_{s}\right)^{\frac{1}{\alpha}}
$$

Summing (15) from $n_{2}$ to $n-1$, we obtain

$$
y_{n} \leq y_{n_{2}}-M_{2} \sum_{s=n_{2}}^{n-1}\left(\frac{1}{a_{s}} \sum_{t=n_{2}}^{s-1} q_{t}\right)^{\frac{1}{\alpha}}
$$

In the last inequality using (13) implies that $y_{n} \rightarrow-\infty$, as $n \rightarrow \infty$ which is a contradiction to the positivity of $y_{n}$. Thus $M=0$, and the proof is complete.

\section{Theorem 3 If}

$$
\sum_{n=n_{0}}^{\infty}\left(\frac{1}{a_{n}} \sum_{s=n_{0}}^{n-1} A^{\alpha}(\sigma(s)) q_{s}\right)^{\frac{1}{\alpha}}=\infty
$$

then (1) oscillates.

Proof: Assume the contrary that $\left\{y_{n}\right\}$ is a solution of (1) such that $y_{n}>0, y_{\sigma(n)}>0$ for all $n \geq n_{1} \geq n_{0}$. Note that (8) is essential for (16) to hold. The function

$$
\sum_{s=n_{0}}^{n-1} A^{\alpha}(\sigma(s)) q_{s}
$$

is unbounded due to (3) and $\Delta A(n)<0,(8)$ must hold. Then, by Lemma 2.1, $\left\{y_{n}\right\}$ satisfies (9) for all $n \geq n_{1}$. It follows from $\left\{\frac{y_{n}}{A(n)}\right\}$ is nondecreasing there is a constant $M>0$ and $n_{2} \geq n_{1}$ such that $y_{n} \geq M A(n)$ for $n \geq n_{2}$. Substituting this inequality into (1), we see that

$$
-\Delta\left(a_{n}\left(\Delta y_{n}\right)^{\alpha}\right) \geq M^{\alpha} q_{n} A^{\alpha}(\sigma(n)) .
$$

Summing (17) from $n_{2}$ to $n-1$, we have 
International Journal of Mathematical, Engineering and Management Sciences

Vol. 4, No. 6, 1459-1470, 2019

https://dx.doi.org/10.33889/IJMEMS.2019.4.6-115

$$
-a_{n}\left(\Delta y_{n}\right)^{\alpha} \geq M^{\alpha} \sum_{s=n_{2}}^{n-1} q_{n} A^{\alpha}(\sigma(s)),
$$

that is,

$$
-\Delta y_{n} \geq \frac{M}{a_{n}^{\frac{1}{\alpha}}}\left(\sum_{s=n_{2}}^{n-1} q_{s} A^{\alpha}(\sigma(s))\right)^{\frac{1}{\alpha}}
$$

Again taking summation from $n_{2}$ to $n-1$ and taking (16) into account, we get

$$
y_{n_{2}} \leq y_{n}-\sum_{s=n_{2}}^{n-1} \frac{M}{a_{s}^{\frac{1}{\alpha}}}\left(\sum_{t=n_{2}}^{s-1} q_{t} A^{\alpha}(\sigma(t))\right)^{\frac{1}{\alpha}} \rightarrow-\infty, \text { as } n \rightarrow \infty,
$$

This contradiction completes the proof.

In the next theorem, we derive the oscillation criteria for the case when (16) does not satisfy.

\section{Theorem 4 If}

$\limsup _{n \rightarrow \infty} A^{\alpha}(\sigma(n)) \sum_{s=n_{1}}^{n-1} q_{s}>1$,

for any $n_{1} \geq n_{0}$, then (1) oscillates.

Proof: Assume the contrary that $\left\{y_{n}\right\}$ is a solution of (1) such that $y_{n}>0, y_{\sigma(n)}>0$ for $n \geq n_{1} \geq n_{0}$. First note that (18) along with (3) imply (8). Then by Lemma 1, $\left\{y_{n}\right\}$ satisfies (9) for all $n \geq n_{1}$. Summing (1) from $n_{1}$ to $n-1$ and applying the fact that $\left\{y_{n}\right\}$ is decreasing, we have

$$
\begin{aligned}
-a_{n}\left(\Delta y_{n}\right)^{\alpha}= & -a_{n_{1}}\left(\Delta y_{n_{1}}\right)^{\alpha}+\sum_{s=n_{1}}^{n-1} q_{s} y_{\sigma(s)}^{\alpha} \\
& \geq y_{\sigma(n)}^{\alpha} \sum_{s=n_{1}}^{n-1} q_{s}
\end{aligned}
$$

Using Lemma 1, we obtain (11), and together with the above inequality leads to

$$
\begin{gathered}
-a_{n}\left(\Delta y_{n}\right)^{\alpha} \geq-a_{\sigma(n)}\left(\Delta y_{\sigma(n)}\right)^{\alpha} A^{\alpha}(\sigma(n)) \sum_{s=n_{1}}^{n-1} q_{s} \\
\geq-a_{n}\left(\Delta y_{n}\right)^{\alpha} A^{\alpha}(\sigma(n)) \sum_{s=n_{1}}^{n-1} q_{s},
\end{gathered}
$$

and hence we obtain

$$
\limsup _{n \rightarrow \infty} A^{\alpha}(\sigma(n)) \sum_{s=n_{1}}^{n-1} q_{s} \leq 1
$$


International Journal of Mathematical, Engineering and Management Sciences

Vol. 4, No. 6, 1459-1470, 2019

https://dx.doi.org/10.33889/IJMEMS.2019.4.6-115

This contradicts (18) and the proof is complete.

Theorem 5 Suppose that condition (8) holds and if there is a positive solution $\left\{\rho_{n}\right\}$ such that

$$
\limsup _{n \rightarrow \infty}\left\{\frac{A^{\alpha}(n)}{\rho_{n}} \sum_{s=N}^{n-1}\left(\rho_{s} q_{s}\left(\frac{A(\sigma(s))}{A(s)}\right)^{\alpha}-\frac{a_{s}}{(\alpha+1)^{\alpha+1}} \frac{\left(\Delta \rho_{s}\right)^{\alpha+1}}{\rho_{s}^{\alpha}}\left(\frac{A(s)}{A(s+1)}\right)^{\alpha(\alpha+1)}\right)\right\}>1
$$

for any $N \geq n_{0}$, then (1) oscillates.

Proof: Assume the contrary that $\left\{y_{n}\right\}$ is a solution of (1) such that $y_{n}>0$, and $y_{\sigma(n)}>0$, for all $n \geq n_{1} \geq n_{0}$. By Lemma 2.1, $\left\{y_{n}\right\}$ satisfies (9) for $n \geq n_{1}$. Define

$$
v_{n}=\rho_{n}\left(\frac{a_{n}\left(\Delta y_{n}\right)^{\alpha}}{y_{n}^{\alpha}}+\frac{1}{A^{\alpha}(n)}\right), \quad n \geq n_{1} .
$$

By virtue of (11), we see that $v_{n} \geq 0$ for all $n \geq n_{1}$. From (20) we have

$$
\Delta v_{n}=\frac{\Delta \rho_{n}}{\rho_{n+1}} v_{n+1}+\rho_{n} \frac{\Delta\left(a_{n}\left(\Delta y_{n}\right)^{\alpha}\right)}{y_{n}^{\alpha}}-\frac{\rho_{n} a_{n+1}\left(\Delta y_{n+1}\right)^{\alpha}}{y_{n}^{\alpha} y_{n+1}^{\alpha}} \Delta y_{n}^{\alpha}+\rho_{n} \Delta\left(\frac{1}{A^{\alpha}(n)}\right) \text {. }
$$

From (1) and (12), we have

$$
\Delta\left(a_{n}\left(\Delta y_{n}\right)^{\alpha}\right) \leq-\left(\frac{A(\sigma(n))}{A(n)}\right)^{\alpha} q_{n} y_{n}^{\alpha}, \quad n \geq n_{2} \geq n_{1} .
$$

Using (22) in (21) one obtains

$$
\begin{aligned}
\Delta v_{n} \leq \frac{\Delta \rho_{n}}{\rho_{n+1}} v_{n+1}-\rho_{n} q_{n}\left(\frac{A(\sigma(n))}{A(n)}\right)^{\alpha}-\rho_{n}\left(\frac{v_{n+1}}{\rho_{n+1}}-\right. & \left.\frac{1}{A^{\alpha}(n+1)}\right) \frac{\Delta y_{n}^{\alpha}}{y_{n}^{\alpha}} \\
& +\rho_{n} \Delta\left(\frac{1}{A^{\alpha}(n)}\right), \quad n \geq n_{2}
\end{aligned}
$$

By Mean Value Theorem,

$$
\Delta y_{n}^{\alpha}=\alpha t^{\alpha-1} \Delta y_{n},
$$

where $y_{n+1}<t<y_{n}$. Since $\Delta y_{n}<0$, we see that

$$
\Delta y_{n}^{\alpha} \leq \alpha \frac{y_{n+1}^{\alpha}}{y_{n}} \Delta y_{n} \text {. }
$$

From (23) and (24), we obtain 
International Journal of Mathematical, Engineering and Management Sciences

Vol. 4, No. 6, 1459-1470, 2019

https://dx.doi.org/10.33889/IJMEMS.2019.4.6-115

$$
\begin{aligned}
\Delta v_{n} \leq-\rho_{n} q_{n}\left(\frac{A(\sigma(n))}{A(n)}\right)^{\alpha}+\frac{\Delta \rho_{n}}{\rho_{n+1}} v_{n+1}-\alpha \rho_{n}\left(\frac{v_{n+1}}{\rho_{n+1}}-\frac{1}{A^{\alpha}(n+1)}\right) \frac{y_{n+1}^{\alpha}}{y_{n}^{\alpha+1}} \Delta y_{n}+\rho_{n} \Delta\left(\frac{1}{A^{\alpha}(n)}\right) \\
\leq-\rho_{n} q_{n}\left(\frac{A(\sigma(n))}{A(n)}\right)^{\alpha}+\frac{\Delta \rho_{n}}{\rho_{n+1}} v_{n+1} \\
-\alpha \rho_{n}\left(\frac{v_{n+1}}{\rho_{n+1}}-\frac{1}{A^{\alpha}(n+1)}\right) \frac{A^{\alpha+1}(n+1)}{A^{\alpha+1}(n)} \frac{\Delta y_{n}}{y_{n+1}^{\alpha}}+\rho_{n} \Delta\left(\frac{1}{A^{\alpha}(n)}\right),
\end{aligned}
$$

where we have used $\left\{\frac{y_{n}}{A(n)}\right\}$ is nondecreasing. Again using $a_{n}^{\frac{1}{\alpha}} \Delta y_{n}$ is decreasing and then from (20), we obtain

$$
\begin{aligned}
\Delta v_{n} & \leq-\rho_{n} q_{n}\left(\frac{A(\sigma(n))}{A(n)}\right)^{\alpha}+\frac{\Delta \rho_{n}}{\rho_{n+1}} v_{n+1} \\
- & \frac{\alpha \rho_{n}}{a_{n}^{\frac{1}{\alpha}}}\left(\frac{A(n+1)}{A(n)}\right)^{\alpha+1}\left(\frac{v_{n+1}}{\rho_{n+1}}-\frac{1}{A^{\alpha}(n+1)}\right)^{1+\frac{1}{\alpha}}+\rho_{n} \Delta\left(\frac{1}{A^{\alpha}(n)}\right) .
\end{aligned}
$$

Let $A=\frac{\Delta \rho_{n}}{\rho_{n+1}}, B=\frac{\alpha \rho_{n}}{a_{n}^{\frac{1}{\alpha}} \rho_{n+1}^{1+\frac{1}{\alpha}}}\left(\frac{A(n+1)}{A(n)}\right)^{\alpha+1}, C=\frac{\rho_{n+1}}{A^{\alpha}(n+1)}$ and using Lemma 6 of Wu et al. (2016), we get

$$
\Delta v_{n} \leq-\rho_{n} q_{n}\left(\frac{A(\sigma(n))}{A(n)}\right)^{\alpha}+\frac{a_{n}}{(\alpha+1)^{\alpha+1}} \frac{\left(\Delta \rho_{n}\right)^{\alpha+1}}{\rho_{n}^{\alpha}}\left(\frac{A(n)}{A(n+1)}\right)^{\alpha(\alpha+1)}+\Delta\left(\frac{\rho_{n}}{A^{\alpha}(n)}\right), \quad n \geq n_{2} .
$$

Summing (25) from $n_{2}$ to $n-1$, we obtain

$$
\sum_{s=n_{2}}^{n-1}\left(\rho_{s} q_{s}\left(\frac{A(\sigma(s))}{A(s)}\right)^{\alpha}-\frac{a_{s}}{(\alpha+1)^{\alpha+1}} \frac{\left(\Delta \rho_{s}\right)^{\alpha+1}}{\rho_{s}^{\alpha}}\left(\frac{A(s)}{A(s+1)}\right)^{\alpha(\alpha+1)}\right)-\frac{\rho_{n}}{A^{\alpha}(n)}+\frac{\rho_{n_{2}}}{A^{\alpha}\left(n_{2}\right)} \leq v_{n_{2}}-v_{n} \text {. }
$$

Using the definition of $v_{n}$ in the above inequality leads to

$$
\begin{aligned}
\sum_{s=n_{2}}^{n-1}\left(\rho_{s} q_{s}\left(\frac{A(\sigma(s))}{A(s)}\right)^{\alpha}-\frac{a_{s}}{(\alpha+1)^{\alpha+1}} \frac{\left(\Delta \rho_{s}\right)^{\alpha+1}}{\rho_{s}^{\alpha}}\right. & \left.\left(\frac{A(s)}{A(s+1)}\right)^{\alpha(\alpha+1)}\right) \\
& \leq \rho_{n_{2}} \frac{a_{n_{2}}\left(\Delta y_{n_{2}}\right)^{\alpha}}{y_{n_{2}}^{\alpha}}-\rho_{n} \frac{a_{n}\left(\Delta y_{n}\right)^{\alpha}}{y_{n}^{\alpha}} .
\end{aligned}
$$


International Journal of Mathematical, Engineering and Management Sciences

Vol. 4, No. 6, 1459-1470, 2019

https://dx.doi.org/10.33889/IJMEMS.2019.4.6-115

On the other hand, from (11), it follows that

$\frac{-\rho_{n}}{A^{\alpha}(n)} \leq \rho_{n} \frac{a_{n}\left(\Delta y_{n}\right)^{\alpha}}{y_{n}^{\alpha}} \leq 0$.

Combining (26) and (27), we get

$$
\begin{gathered}
\sum_{s=n_{2}}^{n-1}\left(\rho_{s} q_{s}\left(\frac{A(\sigma(s))}{A(s)}\right)^{\alpha}-\frac{a_{s}}{(\alpha+1)^{\alpha+1}} \frac{\left(\Delta \rho_{s}\right)^{\alpha+1}}{\rho_{s}^{\alpha}}\left(\frac{A(s)}{A(s+1)}\right)^{\alpha(\alpha+1)}\right) \\
\leq \frac{\rho_{n}}{A^{\alpha}(n)}+\rho_{n_{2}} \frac{a_{n_{2}}\left(\Delta y_{n_{2}}\right)^{\alpha}}{y_{n_{2}}^{\alpha}} .
\end{gathered}
$$

Multiplying (28) by $\frac{A^{\alpha}(n)}{\rho_{n}}$ and then taking lim sup as $n \rightarrow \infty$, we get

$\limsup _{n \rightarrow \infty} \frac{A^{\alpha}(n)}{\rho_{n}} \sum_{s=n_{2}}^{n-1}\left(\rho_{s} q_{s}\left(\frac{A(\sigma(s))}{A(s)}\right)^{\alpha}-\frac{a_{s}}{(\alpha+1)^{\alpha+1}} \frac{\left(\Delta \rho_{s}\right)^{\alpha+1}}{\rho_{s}^{\alpha}}\left(\frac{A(s)}{A(s+1)}\right)^{\alpha(\alpha+1)}\right) \leq 1, \quad$ which contradicts (18). This completes the proof.

By taking $\rho_{n}=A^{\alpha}(n), \rho_{n}=A(n)$, and $\rho_{n}=1$ following corollaries are consequent from Theorem 2.5 , respectively.

Corollary 6 Assume that (4) holds. If

$\underset{n \rightarrow \infty}{\limsup } \sum_{s=N}^{n-1}\left(q_{s} A^{\alpha}(\sigma(s))-\left(\frac{\alpha}{\alpha+1}\right)^{\alpha+1} \frac{A^{\alpha^{2}+\alpha}(s)}{a_{s}^{\frac{1}{\alpha}} A^{\alpha^{2}+\alpha+1}(s+1)}\right)>1$,

for any $N \geq n_{0}$, then (1) oscillates.

Corollary 7 Assume that (4) holds. If

$\underset{n \rightarrow \infty}{\limsup }\left(A^{\alpha-1}(n) \sum_{s=N}^{n-1} q_{s} \frac{A^{\alpha}(\sigma(s))}{A^{\alpha-1}(s)}-\frac{1}{(\alpha+1)^{\alpha+1} a_{s}^{\frac{1}{\alpha}}} \frac{A^{\alpha^{2}}(s)}{A^{\alpha^{2}+\alpha}(s+1)}\right)>1$,

for any $N \geq n_{0}$, then (1) is oscillates.

Corollary 8 Assume that (4) holds. If

$\limsup _{n \rightarrow \infty}\left(A^{\alpha}(n) \sum_{s=N}^{n-1} q_{s}\left(\frac{A(\sigma(s))}{A(s)}\right)^{\alpha}\right)>1$,

for any $N \geq n_{0}$, then (1) is oscillates. 
International Journal of Mathematical, Engineering and Management Sciences

Vol. 4, No. 6, 1459-1470, 2019

https://dx.doi.org/10.33889/IJMEMS.2019.4.6-115

\section{Examples}

This section illustrates the effectiveness of our outcomes having the subsequent difference equations.

Example 1. Consider the second order advanced type difference equation

$\Delta\left(n^{\frac{5}{3}}(n+1)^{\frac{5}{3}}\left(\Delta y_{n}\right)^{\frac{5}{3}}\right)+n^{2} y_{2 n}^{\frac{5}{3}}=0, \quad n \geq 1$,

Here $a_{n}=n^{\frac{5}{3}}(n+1)^{\frac{5}{3}}, q_{n}=n^{2}, \sigma(n)=2 n$, and $\alpha=\frac{5}{3}$, and

$$
A(n)=\sum_{s=n}^{\infty} \frac{1}{s(s+1)}=\frac{1}{n} \text {. }
$$

Clearly the conditions (3) and (8) are satisfied, and the condition (13) becomes

$$
\sum_{n=1}^{\infty}\left(\frac{1}{n^{\frac{5}{3}}(n+1)^{\frac{5}{3}}} \sum_{s=1}^{n-1} s^{2}\right)^{\frac{3}{5}} \approx \sum_{n=1}^{\infty} \frac{1}{n^{\frac{1}{5}}}=\infty .
$$

Then by Theorem 5 any nonoscillatory of (32) converges to zero as $n \rightarrow \infty$.

Example 2. Consider the following difference equation

$$
\Delta\left(n^{\frac{1}{3}}(n+1)^{\frac{1}{3}}\left(\Delta y_{n}\right)^{\frac{1}{3}}\right)+n^{\frac{4}{3}} y_{2 n}^{\frac{1}{3}}=0, \quad n \geq 1,
$$

Here $a_{n}=(n(n+1))^{\frac{1}{3}}, \quad q_{n}=n^{\frac{4}{3}}, \sigma(n)=2 n$, and $\alpha=\frac{1}{3}$. By simple calculation, we obtain

$$
A(n)=\sum_{s=n}^{\infty} \frac{1}{a_{n}^{\frac{1}{\alpha}}}=\sum_{s=n}^{\infty} \frac{1}{s(s+1)}=\frac{1}{n} .
$$

The conditions (3) and (8) are clearly satisfied and the condition (16) becomes

$$
\sum_{n=1}^{\infty} \frac{1}{n(n+1)}\left(\sum_{s=1}^{n-1} \frac{1}{(2 s)^{\frac{1}{3}}} s^{\frac{4}{3}}\right)^{3} \sum_{n=1}^{\infty} \frac{n^{2}(n-1)^{3}}{2^{\frac{4}{3}}(n+1)}=\infty .
$$

Then by Theorem 6, every solution of (33) is oscillatory.

Example 3. Consider the following difference equation

$\Delta\left(n(n+1) \Delta y_{n}\right)+\lambda y_{2 n}=0, \quad n \geq 1, \lambda>0$.

Here $a_{n}=n(n+1), q_{n}=\lambda, \sigma(n)=2 n, \alpha=1$, and 
International Journal of Mathematical, Engineering and Management Sciences

Vol. 4, No. 6, 1459-1470, 2019

https://dx.doi.org/10.33889/IJMEMS.2019.4.6-115

$$
A(n)=\sum_{s=n}^{\infty} \frac{1}{s(s+1)}=\frac{1}{n}
$$

The conditions (3) and (8) are clearly satisfied and the condition (18) becomes

$$
\lim _{n \rightarrow \infty} \sup \frac{1}{2 n} \sum_{s=1}^{n-1} \lambda=\lim _{n \rightarrow \infty} \sup \frac{\lambda(n-1)}{2 n}=\frac{\lambda}{2} \text {. }
$$

If $\lambda>2$, then by Theorem 7 , every solution of equation (34) is oscillatory.

\section{Conclusion}

The results presented in this paper are new and of high degree of generality. The results obtained in the literature (Zhang and Li, 1998; Ping and Han, 2003; Arul and Ayyappan, 2013) are applicable only when the studied equation is linear and canonical, but the results presented in this paper are applicable to linear and half-linear equations with noncanonical forms. Therefore the results provided in this paper complement, extend and enhance the existing outcomes recorded in the literature. Further three examples are provided to dwell upon the importance of our main results.

It might also be interesting to extend the results of this paper to higher order advanced type difference equation

$$
\Delta\left(a_{n}\left(\Delta^{m-1} y_{n}\right)^{\alpha}\right)+q_{n} y_{\sigma(n)}^{\beta}=0, \quad n \geq n_{0},
$$

where $m \geq 2$ is an even integer and $\alpha$ and $\beta$ are ratio of odd positive integers.

\section{Conflict of Interest}

The authors confirm that this article contents have no conflict of interest.

\section{Acknowledgements}

The authors thank the reviewer for his/her valuable suggestions and corrections that improved the contents of the paper.

\section{References}

Agarwal, R.P. (2000). Difference equations and inequalities: theory, methods, and applications. CRC Press. ISBN: 9780824790073.

Agarwal, R.P., Bohner, M., Grace, S.R., \& O'Regan, D. (2005). Discrete oscillation theory. Hindawi Publishing. Corporation., New York.

Arul, R., \& Ayyappan, G. (2013). Oscillation theorems for second order neutral delay and advanced difference equations. International Journal of Differential Equations and Applications, 12(1), 13-25.

Elsgolts, L.E., \& Norkin, S.B. (1973). Introduction to the theory and applications of differential equations with deviating arguments (Vol. 105). Academic Press, New York.

Ocalan, O., \& Akin, O. (2007). Oscillatory property for advanced difference equations. Novi Sad Journal of 
International Journal of Mathematical, Engineering and Management Sciences

Vol. 4, No. 6, 1459-1470, 2019

https://dx.doi.org/10.33889/IJMEMS.2019.4.6-115

Mathematics, 37(1), 39-47.

Ping, B., \& Han, M. (2003). Oscillation of second order difference equations with advanced arguments. Conference Publications, American Institute of Mathematical. Sciences, 108-112.

Selvarangam, S., Geetha, S., Thandapani, E., \& Pinelas, S. (2016). Classifications of solutions of second order nonlinear neutral difference equations of mixed type. Dynamics of Continuous, Discrete and Impulsive Systems Series B: Applications \& Algorithms, 23(6), 433-447.

Thandapani, E., Ravi, K., \& Graef, J.R. (2001). Oscillation and comparison theorems for half-linear second order difference equations. Computers \& Mathematics with Applications, 42(6-7), 953-960.

Trench, W.F. (1974). Canonical forms and principal systems for general disconjugate equations. Transactions of the American Mathematical Society, 189, 319-327.

Wu, H., Erbe, L., \& Peterson, A. (2016). Oscillation of solution to second-order half-linear delay dynamic equations on time scales. Electronic Journal of Differential Equations, 2016(71), 1-15.

Zhang, B., \& Cheng, S.S. (1995). Comparison and oscillation theorems for an advanced type difference equation. Annals of Differential Equations, 1995(4), 485-494.

Zhang, Z., \& Li, Q. (1998). Oscillation theorems for second-order advanced functional difference equations. Computers \& Mathematics with Applications, 36(6), 11-18.

Zhang, Z., \& Zhang, J. (1999). Oscillation criteria for second-order functional difference equations with "summation small” coefficient. Computers \& Mathematics with Applications, 38(1), 25-31. 